\title{
Electron microscopy for the characterisation of atmospheric particles
}

\author{
P.A. Buffat \\ CIME, École Polytechnique Fédérale de Lausanne, CH-1015 lausanne, Switzerland
}

\begin{abstract}
Transmission and scanning electron microscopy and their analytical associated techniques constitute an efficient way to assess the presence and to identify particles in the range of the micrometer and down to the nanometer even when the constitute only a low fraction of the total dust. Recent developments in high resolution transmission electron microscopy and electron energy loss spectroscopy may lead to new means of characterisation of imperfect structures like carbon blacks and soot.
\end{abstract}

\footnotetext{
T
} he impact of fast electrons on matter lead to numerous interactions that provide a rich information about the nature of the target (Fig. 1). Scanning and transmission electron microscopes behave a high resolving power suitable to study fine particles down to the range of the nanometer. The information is got essentially through a particle by particle observation. Such an approach constitutes at the same time the force and weakness of the technique. On one hand the SEM and TEM analysis may require (much) more time than some other techniques which bring data on a full set of particles at a time (X-ray diffraction, infrared spectroscopy, Raman diffusion...). On the other hand, even in the case of low concentration, scarce particles of a particular type can be found, identified and entirely characterised among numerous others by a careful screening of the sample. Moreover, this analysis do not require any model of the statistical distribution as it is the case for the deconvolution process of the raw data in global methods.

Both scanning electron microscope (SEM) and transmission electron microscope (TEM) combine an imaging mode bringing morphological characterisation (shape, size, roughness...) and an analytical mode for chemical information (elemental analysis by X-ray energy dispersive spectroscopy EDS). Electron diffraction in TEM leads to crystallographic structure and phase identification. These means are routinely used for example to assess the presence of asbestos or other inorganic pollutants in professional or remote environment. During the last years, some efforts have been made to try to differentiate carbonaceous material like ashes and soots by high resolution transmission microscopy TEM. The recent development of electron energy loss spectrometry (EELS) in the TEM allows nowadays to distinguish between different valence or bonding states of atoms and may lead to a new approach to describe these materials and to assess their origin.

\section{Scanning electron microscopy}

Scanning electron microscopy is a fast and efficient way to observe and analyse particles, aerosols and airborne materials on filters or substrates as deposited. Most samples require only a light coating with a carbon or metal film to insure electrical conductivity.

The secondary electron (SE) mode brings the morphological information and X-ray energy dispersive spectroscopy (EDS) gives qualitative or even more or less quantitative elemental analysis depending the size of the particle. The combination of morphological and chemical information is often sufficient to identify unambiguously the nature of mineral or inorganic particles. However the absence of crystallographic information may hinder to distinguish between phases with close compositions. It will happen for example 


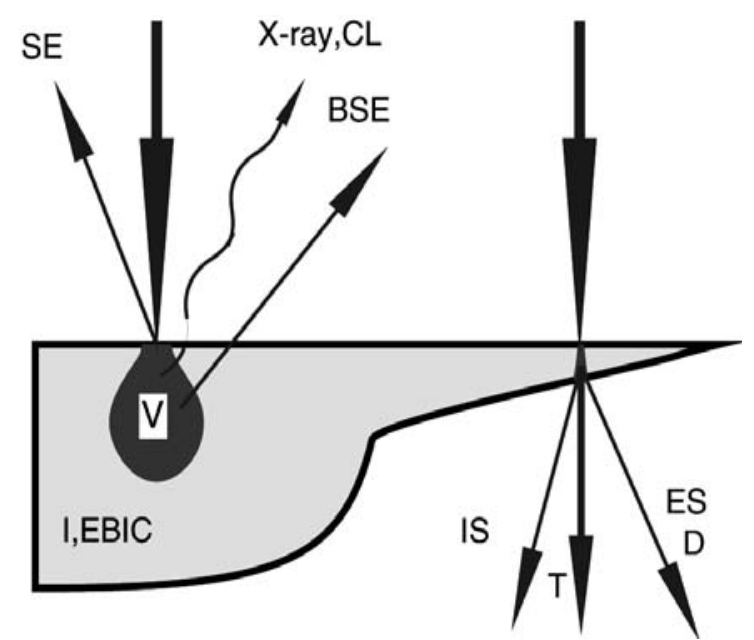

Figure 1. Several interactions arise between the sample and the electron beam. In SEM, the emission of secondary electrons SE, backscattered electrons BSE or X-rays is most often recorded. Sometimes, cathodoluminescence $\mathrm{CL}$ and and absorbed current (I, EBIC) create also a specific contrast. Multiple interactions lead to a volume of excitation $V$. This volume is much smaller in the thin samples used in TEM. Although the same signals are available, the most interesting information is carried by the transmitted electrons $T$, the diffracted electrons $D$, the elastic ES and inelastic IS scattered electrons.

in asbestos assessment where rolled vermiculite behave the same composition than palygorskite fibers (Fig. 3).

The combination of secondary (SE) and backscattered (BSE) electron contrast mode may be useful to distinguish rapidly the relevant particles from the background. For example, Ormstad et al. [1] studied by this way the presence and spreading of cat allergen responsible of asthma and respiratory symptoms in houses. Dust was collected and then marked with $30 \mathrm{~nm}$ gold particles by immuno-labelling to demonstrate the presence of cat (Fe1 d 1) allergen at the surface of the particles. In BSE contrast mode, the gold nanocrystals exhibit a strong contrast and showed that the allergen was present in particles as small as $1 \mu \mathrm{m}$ (Fig. 4).

The nominal resolution of the SEM ranges between 1 and $5 \mathrm{~nm}$ in SE mode. However, under the practical condition of observation for screening such samples, the contrast far from the optimum used for resolution test and the noise of the image may be high, in particular with a fast scanning speed. Thus the useful resolution power is worse by a factor of at least two in slow scan and even 20 at video rate. Moreover it should be realised that in order to recognise an object by its morphology, arrays of several tens of pixels are required. For these reasons, the search for particles of less than one micron in length or diameter becomes difficult and rapidly tiring. In that respect, the use of SEMs fitted with field emission guns much brighter than the conventional thermionic guns ( $\mathrm{W}, \mathrm{LaB}_{6}$ cathodes) improves not only the

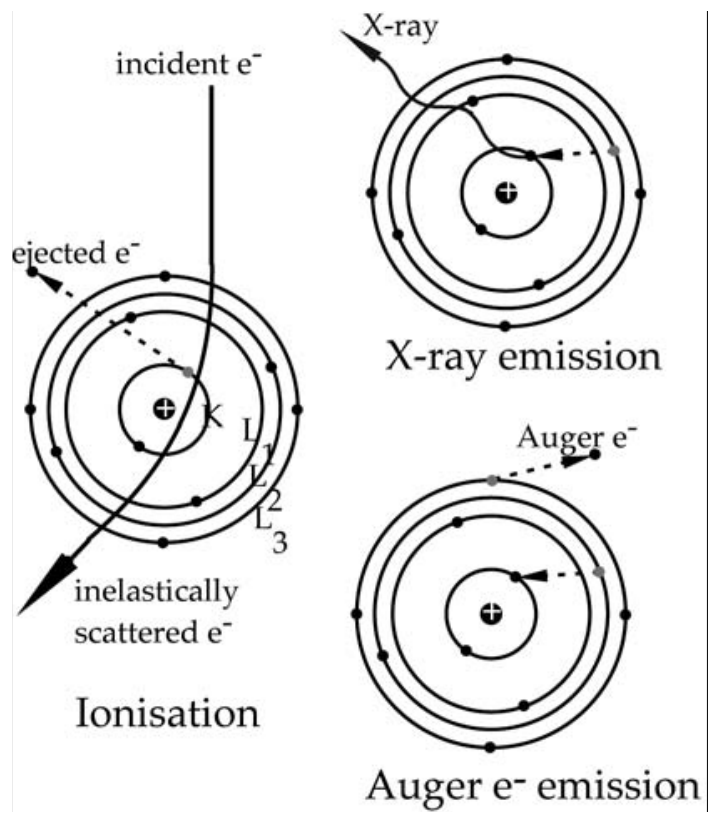

Figure 2. The interaction of an incident electron with the core electrons may ionise the target atom by ejection of an electron. When an electron from an outer shell fills the vacancy, its excess energy is released either by emission of an X-ray photon or of an Auger electron. This latter process is predominant for low energies (ionisation of the $\mathrm{K}$ shell of light elements for example).

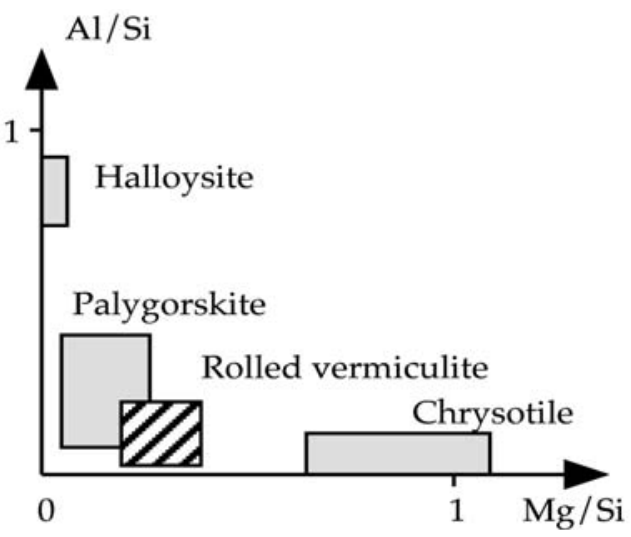

Figure 3. Chemical composition of four phyllosilicates with fibre habit and apparent tubular structure. The composition overlap between palygorskite and rolled vermiculite requires electron diffraction for an unambiguous identification.

resolving power, but also brings much comfort and efficiency to the operator.

For chemical contrast with BSE and for EDS microanalysis, the volume of emission of the signal ranges 

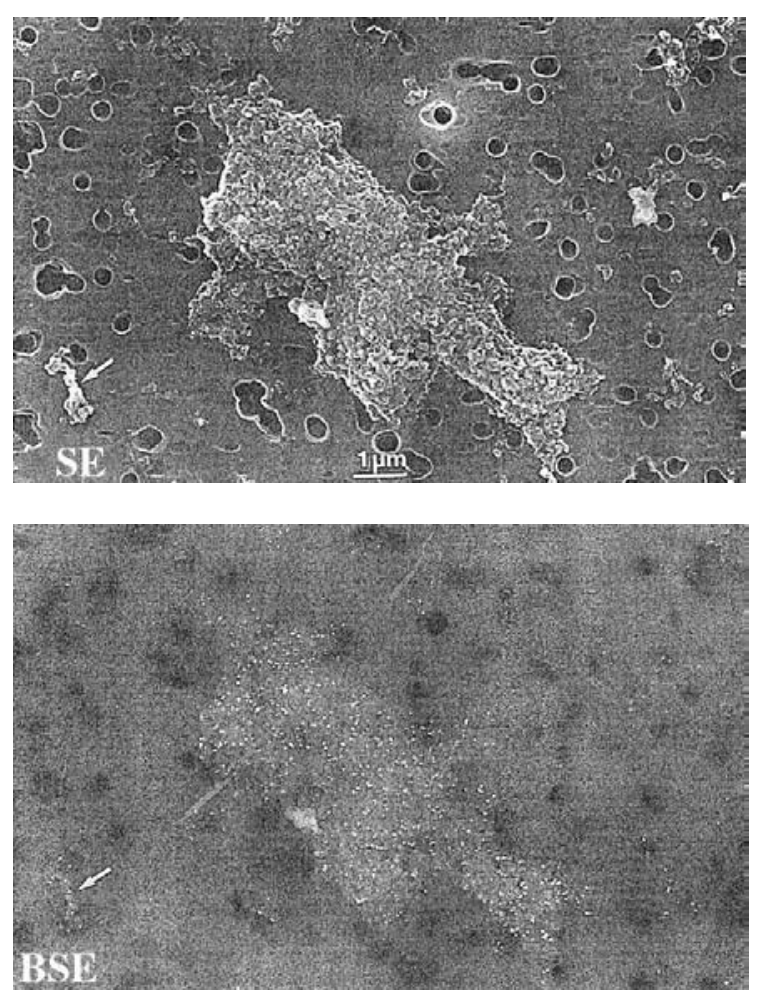

Figure 4. Dust containing immuno-labelled allergen of cat. Top: SE morphological contrast. Bottom: BSE mode showing with high contrast the $30 \mathrm{~nm}$ gold particles of the marker.

between a fraction of a $\mu \mathrm{m}$ and some microns depending of the atomic number of the target et the accelerating voltage.

\section{Sampling and sample preparation}

Electron microscopes work under high vacuum with the exception of the "environmental SEM" which accept gas pressure of a few tens of mbar around the sample. Observation takes a few second at least. Thus particle cannot rest in atmosphere, but must lie on a suitable substrate. Most often, SEM observation is carried out on bulk samples, but information is only got from the surface layer. Only the uppermost nanometers give the morphological information and chemical information comes from a layer of about a micrometer in thickness. Thus there is a risk that the chemical information escape from overlapping particles. TEM samples must in any case be electron transparent, i.e. their thickness should not exceed some tenths of micrometer.

Sampling is most often done by filtering the air on a suitable membrane like polycarbonate or cellulose esther (nitrocellulose) filters or even metallic membranes. The choice of the pore size rely on the particle diameter, although particles smaller than the pore size can be partly retained by impact on the filter surface. When a quantitative assessment of the particle air content is required, a control of the efficiency of filtering is mandatory, for example by mounting two equivalent filter heads in cascade to monitor the amount of particles flying through the first one onto the second one.

For some applications, filtering through a membrane is sometimes replaced by thermophoretic sampling (in flames for example [2]), drifting in an electric field or a preliminary sorting may be performed in sampling heads using the properties of the aerodynamics forces to separate particles of different shape and mass ("cascade impacters" [3]).

Filters containing less than a monolayer of particulate matter are suitable for direct observation in the SEM after coating with a thin conductive layer (most often carbon, sometimes gold or gold-palladium). They can also be prepared as a replica by a "direct transfer method" for TEM investigation on a thin carbon film supported by a copper mesh [4]. The polycarbonate filters are preferred because their flat surface.

The direct observation of the filter in SEM or TEM is suitable as long as the interesting particulate matter represent an important part of the total amount of retained material. This is mostly the case in professionnal exposure to contaminants for example. In remote exposure or general environment, the hunted particles may be present in very small quantities among large quantities of other material, in particular organic one. As long as they are inorganic, their apparent concentration may be increased after sampling by ashing the filter and the organic material in a low temperature oxygen plasma and by redispersing the ashes on a carbon film ("indirect method" [4]).

\section{Transmission electron microscopy}

Transmission electron microscopy requires more preparation effort to get electron transparent samples, but it offers several important advantages in comparison with SEM:

- higher signal to noise ratio and resolution for observation,

- reduced excitation volume to about the probe size (1 $10 \mathrm{~nm}$ ) and the sample thickness,

- diffraction contrast and crystallographic information.

\section{Observation of morphology, counting particles...}

Most often, observation are performed in bright field with the transmitted unscattered electrons. The resolving power of a conventional TEM for samples thin enough is around $3 \AA$ or even below $2 \AA$ for high resolution ones. Even close to the resolution limit, the signal to noise ratio of the image collected in one second or less stays very large and the morphological study of particles with only a few tens of nanometers in diameter is straightforward. Sometimes, diffracted electrons, elastically or inelastically scattered electrons are also used to get a contrast associated to particular 

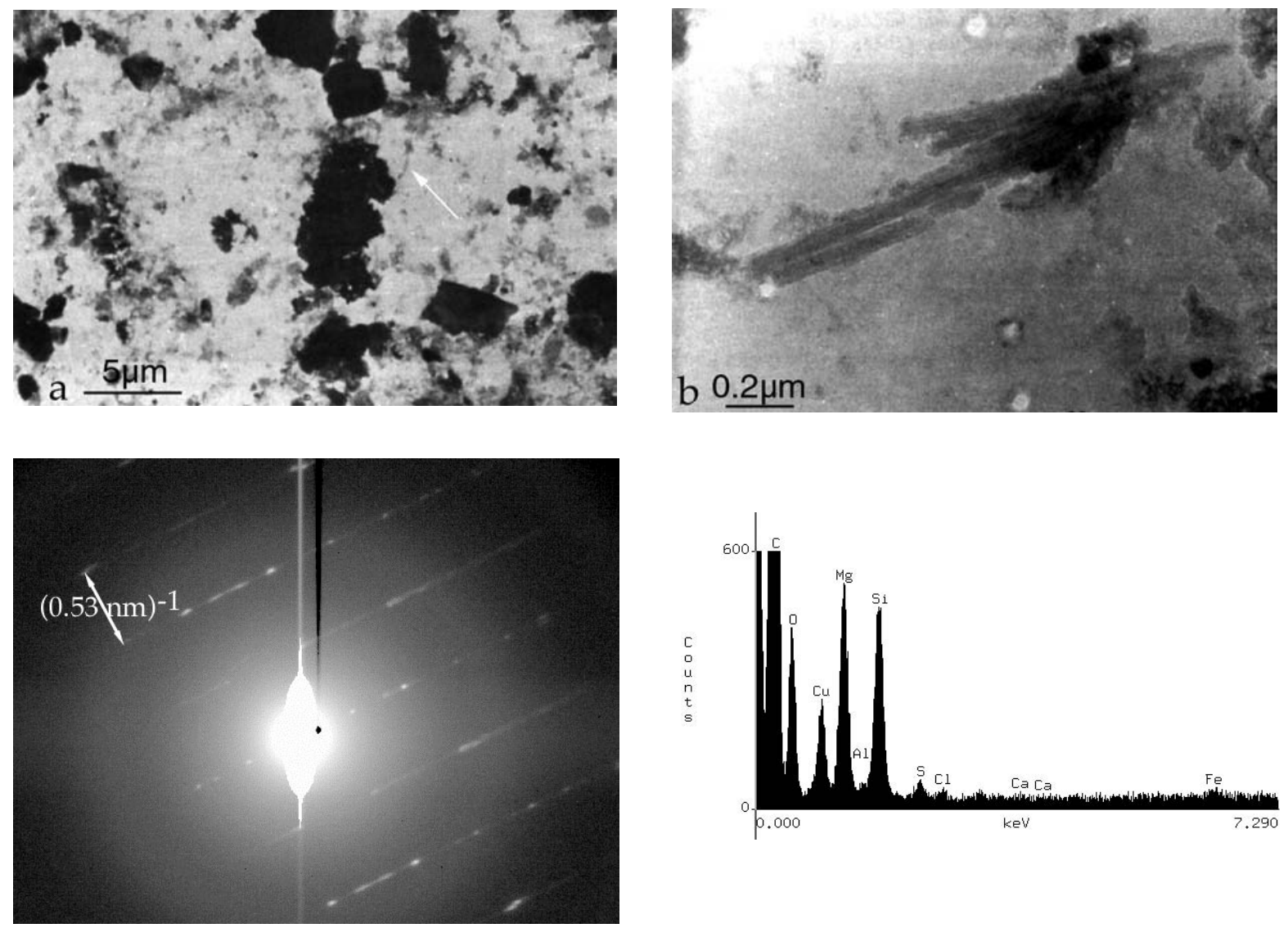

Figure 5. Inorganic particles from urban environment filtered on a $\mathbf{0 . 4 5} \mu \mathrm{m}$ porosity cellulose ester filter and indirectly transferred on a thin carbon membrane for TEM observation after ashing: a) at low TEM magnification the presence of several kind of particles or deposits is obvious, nevertheless the detection of small fibers (arrow) among them is immediate; b) higher magnification confirms the fiber habit. In the bundle c) the diffraction pattern with rows of reflections spaced by $(0.53 \mathrm{~nm})^{-1}$ and with alternate streak and spot structure is characteristic of chrysotile fibers with their axis close to a direction normal to the incident electron b

e

m

d) X-ray EDS spectra confirming the presence of partly degraded chrysotile: the Mg/Si ratio is close to unity, a weak iron peak is present, no or low $\mathrm{Ca}$, the traces of $\mathrm{S}$ and $\mathrm{Cl}$ come from surface degradation, the large copper peak is due to the supporting copper grid hitted by stray or backscattered electrons and X-rays scattered at the condensor aperture level. The carbon peak comes from the supporting carbon film and the peak close to the energy scale origin correspond to electronic noise.

crystallographic conditions or to the target atomic number, or even to a particular chemical element (see EELS underneath).

\section{Crystallography}

Electron diffraction is available in selected area mode on areas as small as $0.2 \mu \mathrm{m}$ in diameter or in microdiffraction mode under probes of a few nanometers in diameter. It lead to the determination of atomic plane spacings and angles in a particle, an additional information to the EDS chemical analysis which removes the ambiguity between phases with close composition.

Crystallographic information is also available from "high resolution (transmission) electron microscopy" HR(T)EM images. In that observation mode, interference fringes produced by the directly transmitted beam and diffracted beams mimic the atomic lattice when the particle is thin enough. Besides the assessment of the lattice for phase identification, HRTEM is particularily useful to describe disorder in poorly or imperfectly crystallised materials, like for example in several forms of carbon and soot.

Such information may be used to relate the structure to the origin or the history of a specific particle, in particular its thermal history, at the difference of the global methods like Raman spectroscopy or X-ray diffraction which require a large set of particles for volume analysis. It may be used later to recognise the origin of a particle among a set of possible scenarios. As example, figure 6 shows the evolution with the treatment temperature of carbon blacks produced by 

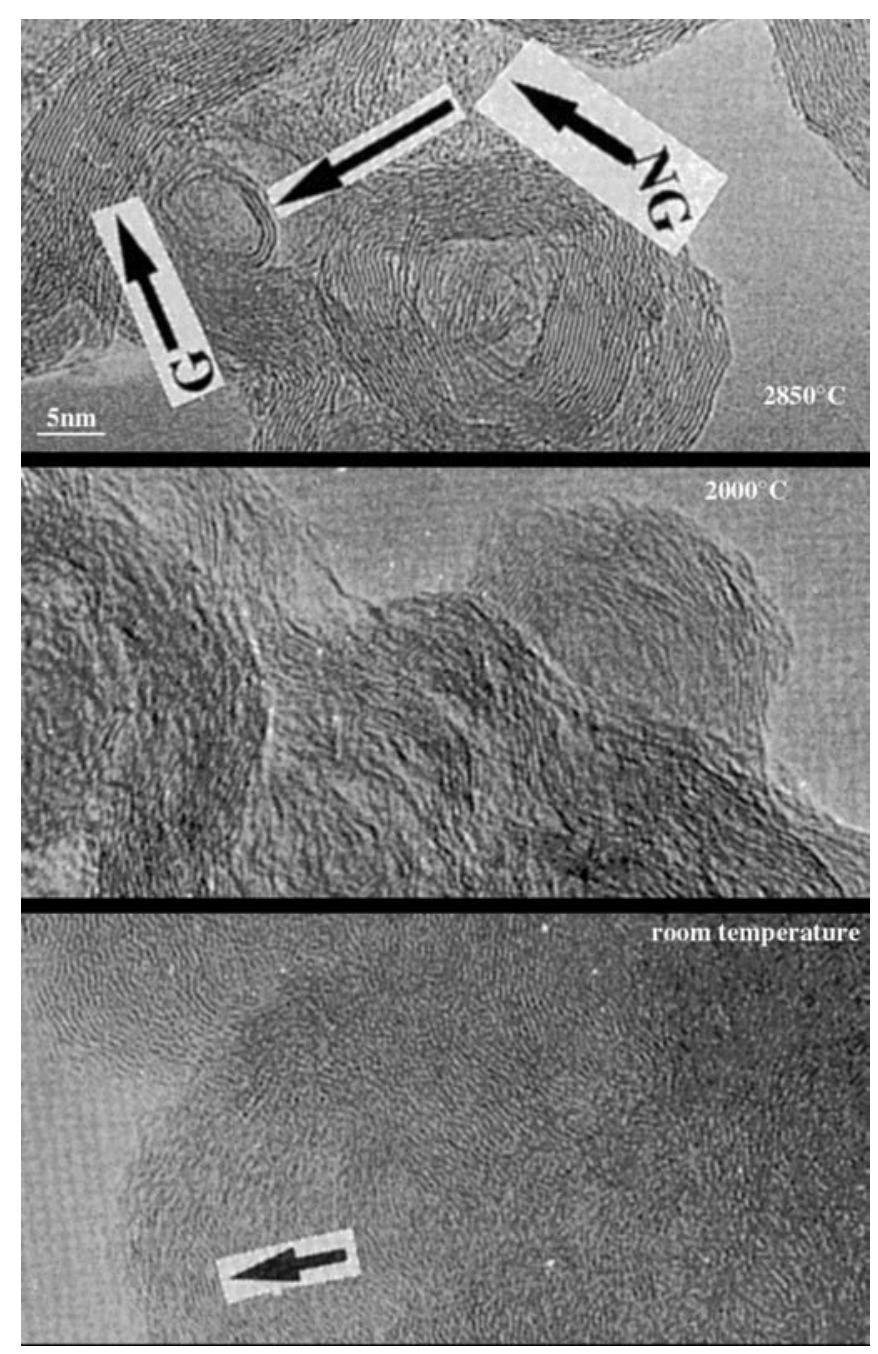

Figure 6. Influence of heat treatment on the crystallinity of carbon black. These pictures were obtained on the same material after heat treatment at $2850{ }^{\circ} \mathrm{C}$ (graphitized (G) faceted areas and non graphitized (NG) pockets), at $2000{ }^{\circ} \mathrm{C}$ (original grain structure with enhanced graphitic planes) and without treatment (arrow: low graphitic order).

$\mathrm{CO}_{2}$ catalytically driven laser pyrolysis of benzene [5]. From untreated to $2000{ }^{\circ} \mathrm{C}$ annealed particles, the original grain structure is retained and only the organisation of the graphitic planes growth. Then between $2000{ }^{\circ} \mathrm{C}$ and $2850{ }^{\circ} \mathrm{C}$, grains sinter and polyhedral rings of well developped graphitic planes appears around pockets with low organisation. Figure 7 and table I illustrate the result of quantitative image analysis on HRTEM pictures of a carbon black particle and a diesel soot particle [6]. Both exhibit a turbostractic structure, with short planes. Most parameters defined in [6] like circularity, elongation, length and fractional coverage which refers to the shape of these planes and do not change from one type of material to the other within
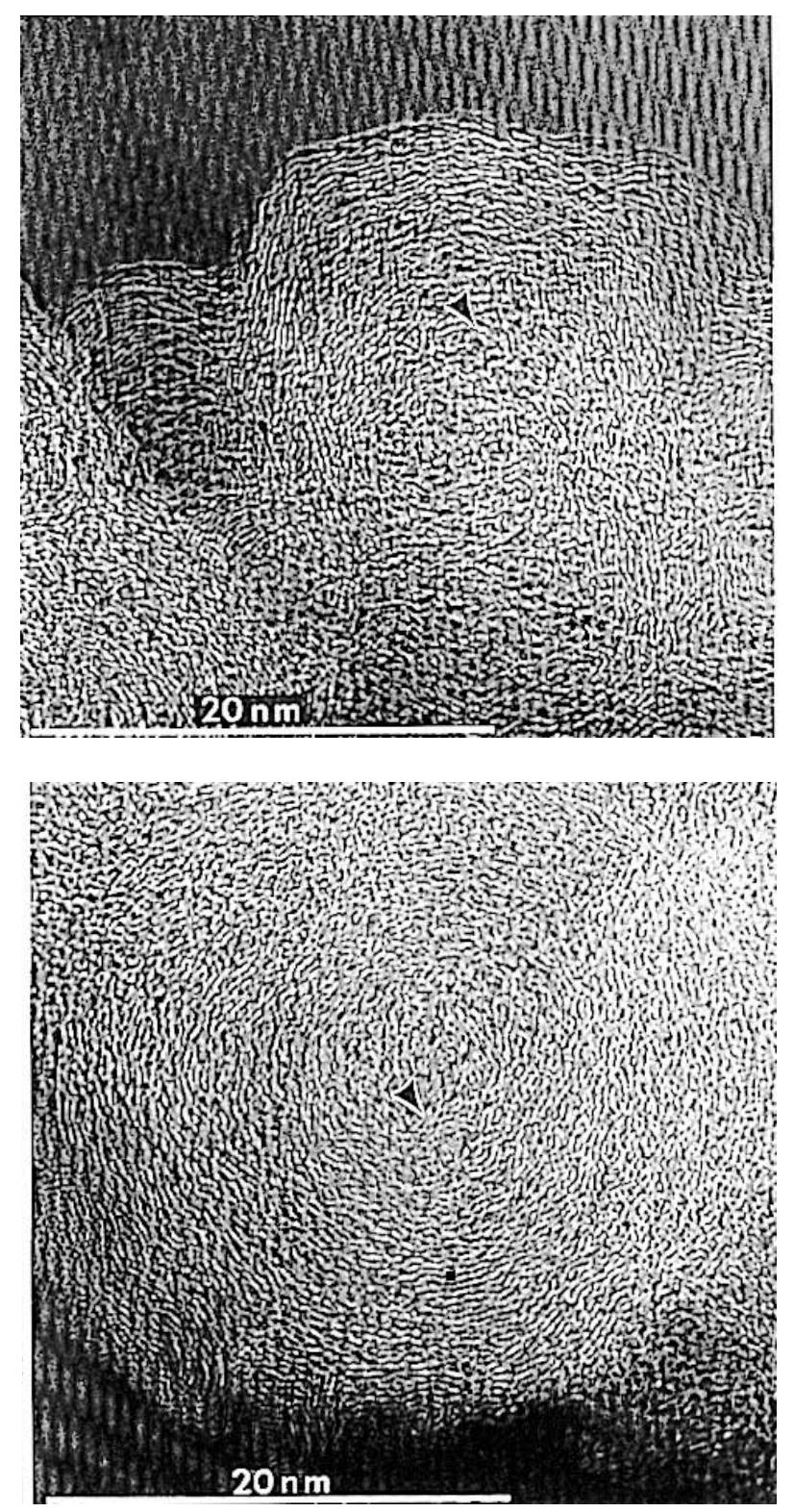

Figure 7. Comparison of the atomic stacking in a carbon black and a diesel soot. Careful analysis of the partial atomic planes and their turbostratic behaviour may lead to some criteria for differentiation: a) carbon black, b) diesel soot.

the experimental accuracy, but the interplanar spacing is significantly different.

\section{EDS and EELS chemical microanalysis}

Like in SEM, X-ray EDS spectroscopy provides chemical information as elemental concentration with a detectability of about $1 \%$. The most recent TEMs fitted with field emission guns provide probes as thin as one nanometer containing still enough current to get an analyse with a counting 
Table I. Structural data of carbon black and diesel soot

\begin{tabular}{lcc}
\hline & Carbon black & Diesel soot \\
\hline Circularity & $0.35 \pm 0.14$ & $0.35 \pm 0.15$ \\
Elongation & $4.3 \pm 2.0$ & $4.3 \pm 2.1$ \\
Length & $11.8 \pm 6.2$ & $12.5 \pm 7.2$ \\
Orientation & $89^{\circ}+41^{\circ}$ & $88^{\circ} \pm 34^{\circ}$ \\
Interplanar spacing & $3.5 \pm 0.1 \AA$ & $3.3 \pm 0.3 \AA$ \\
Fractional coverage & $26.2 \pm 0.5 \%$ & $29.5 \pm 0.6 \%$ \\
\hline
\end{tabular}

time of about 50 seconds. They are the only tool available at present to identify single nanometer-sized particles.

The transmission microscope can also be fitted with an energy filter to measure the energy loss that transmitted electrons suffered when they went through the sample (Fig. 8). Several kind inelastic interaction can be observed and quantified. Among them, the excitation of plasmon losses gives information about the collective oscillations of the electron gas and lead to the electronic properties of the target. Besides their interest to estimate the thickness of the sample, they are often used as fingerprint to recognise different structures or atom bonding in targets of similar or close composition. Ionisation edges corresponding to the energy lost to ionise one target atom are by far the most important information for chemical analysis. They are superimposed

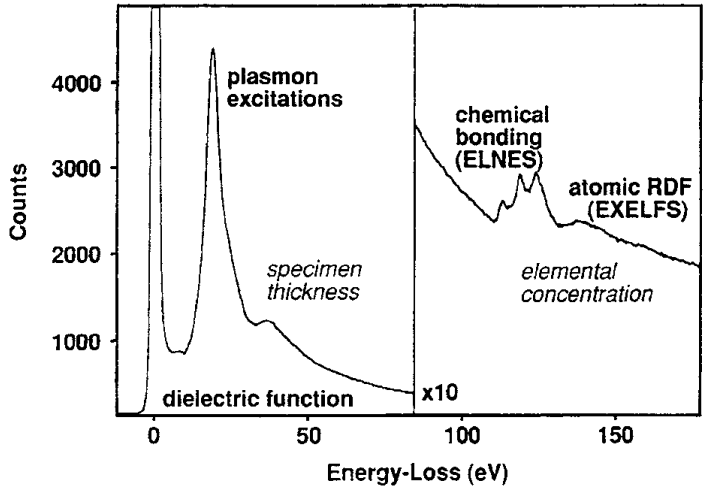

Figure 8. Electron energy loss spectrum. The high peak at left is the "elastic peak" and correspond to electrons which did not interact with the sample or did elastic interaction only. It constitutes with the plasmon peak(s) the "low loss" region. The fast decay of the counts (intensity) requires a change in the detection sensitivity ( $\times 10$ or more) to observe the intensity in the "core loss" region where the ionisation edges are superimposed on a rapidly decreasing background.

on a background which intensity decreases rapidly as a power law of the energy loss. The position of the edge identifies the element, its intensity above the background the concentration. This information is in principle equivalent to that of EDS, but with several advantages:
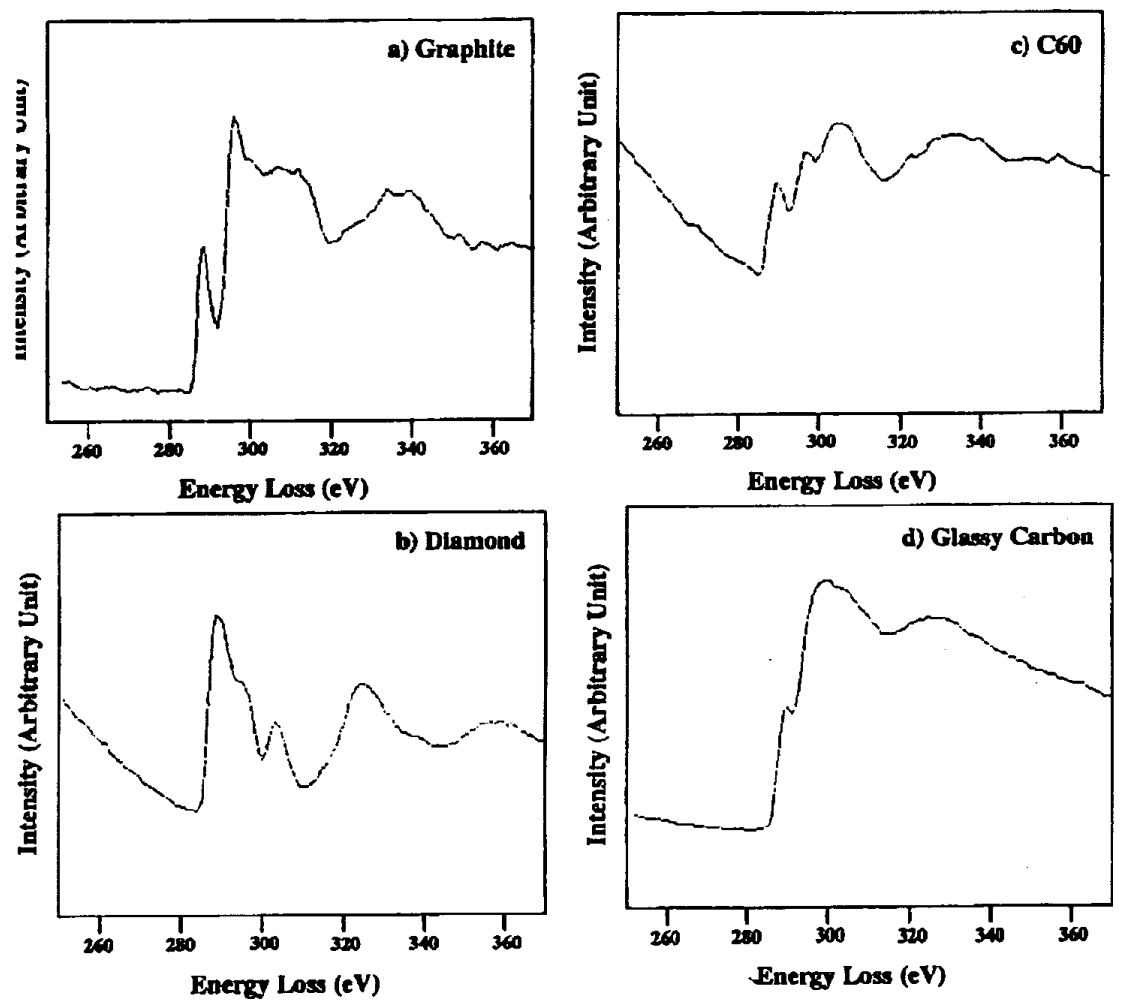

Figure 9. Core losses of EELS spectra (C$\mathrm{K}$ edge) for different forms of carbon [7]. 


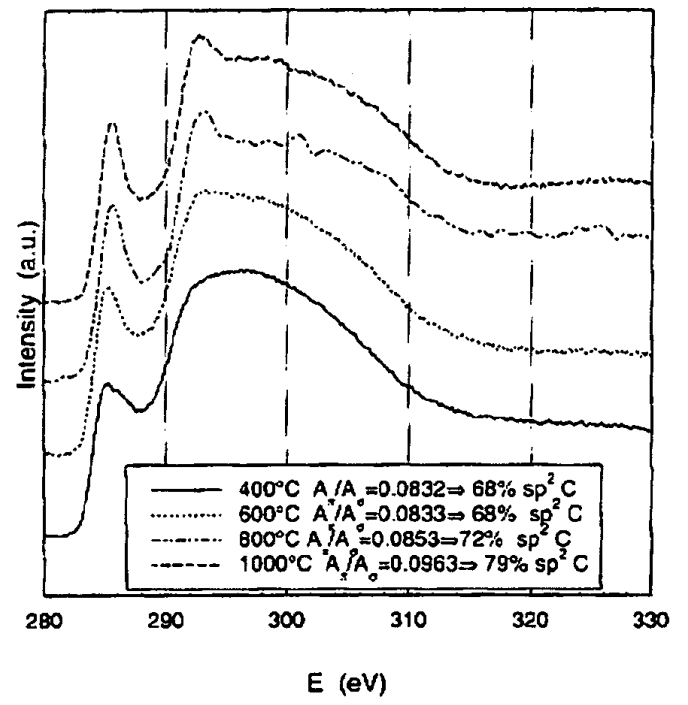

Figure 10. Core loss spectra of pyrolised cellulose at different temperature. Interpretation of the edge profile lead to the $\mathbf{s p}^{2} / \mathbf{s p}^{3}$ carbon content (spectra are slightly shifted in energy for clarity).

- the efficiency of collection of the forward scattered electron is about 100 times higher than that of X-rays and the analysis time takes only $10 \mathrm{msec}$ to a few seconds depending the task,

- all the ionization events are detected even for light elements (which is not the case for EDS due to the prominent Auger process for low Z),

- losses are measured in a range of 0 to about $2000 \mathrm{eV}$ which correspond to the ionisation of the atom outer shells and are thus sensitive to atom bonding.

With the appropriate filters, EELS is also able to provide within a few seconds images of the distribution of an element by accepting only the electrons which suffered the corresponding edge loss.

The fine structure around the edge onset (electron near edge structure ELNES) brings information on the valence state or bonding, although today the means for calculation of ELNES profile are still under development and most of the information is got by comparison between profiles (fingerprints) measured on different compounds (Fig. 9). The post-edge fine structure (extended energy loss fine structure EXELFS, similar to EXAFS for X-rays) gives also information on the atomic environment of the ionised atom (radial distribution function RDF, symmetry).

The ability of EELS to derive information on bonding or atom environment will probably also play an important role

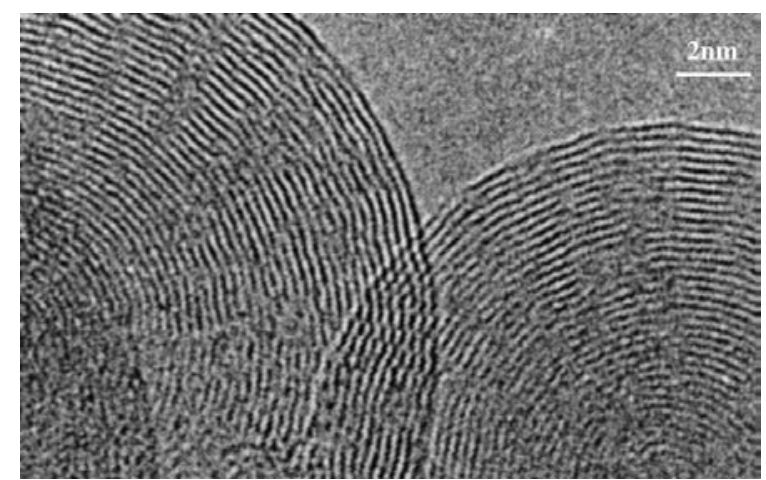

Figure 11. Bucky onions prepared by strong irradiation of amorphous carbon in a TEM.

in the characterisation of imperfect structures like that of carbonaceous contaminants. In particular, analysis of the microstructure by HRTEM like in figure 7 will be replaced, or at least complemented, by EELS measurement. For example, the EELS spectra from figure 10 were recorded on pyrolized cellulose at different temperature and constitute an interesting example of the potential of that technique [8]. Interpreting the low and core losses allow to quantify the content of carbon in $\mathrm{sp}^{2}$ state with respect to $\mathrm{sp}^{3}$. Low losses lead also to the imaginary part of the dielectric function and to optical absorption. These values are important to calculate the absorption of small particles by the Mie theory and may bring some potential explanation for intriguing absorption bands observed in the interstellar space. Moreover the UV absorption at $264 \pm 3 \mathrm{~nm}$ bucky carbon onions (Fig. 11) which were obtained by transformation of amorphous carbon under a strong electron beam or heat treatment of pure carbon soot exhibit several features which closely resemble to the interstellar absorption band at $217 \mathrm{~nm}$ [9].

\section{References}

1. Ormstad, H. et al., J. Immunolog. Meth. 1995, 187, 245-251.

2. Megaridis, C. M.; Dobbins R. A. Twenty Second Symposium on Combustion, The Combustion Institute, Pittsburgh, 1998, 353-362.

3. Vigliani, E. C. et al. Med. Lavoro 1976, 67, 551-567.

4. Steen., D. Détermination du taux de contamination de l'air par l'amiante, EPFL report, 1982 available from philippe.buffat@epfl.ch. See also the recommendations from the ISO.

5. Xiang-Xin, B. et al. J. Mater. Res. 1995, 10, 2875-2884.

6. Palotás, A. B. et al. Microsc. Res. Techn. 1996, 33, 266-278.

7. Kiyoshi, Y. et al. Thin Solid Films 1996, 273, 222-224.

8. Jäger, C.; Mutschke, H.; Henning Th. Astron. Astrophys. 1998, 332, 291-299.

9. de Heer, W. A., Ugarte, D. Chem. Phys. Lett. 1993, 207, 480486. 